\title{
KELT: A Wide-Field Survey of Bright Stars for Transiting Planets
}

\author{
Robert J. Siverd ${ }^{1}$, Joshua Pepper ${ }^{2}$, Kris Stanek ${ }^{1}$, Richard W. Pogge ${ }^{1}$, \\ B. Scott Gaudi ${ }^{1}$ \& Darren L. DePoy ${ }^{1}$ \\ ${ }^{1}$ The Ohio State University \\ $140 \mathrm{~W} 18$ th Ave. \\ Columbus $\mathrm{OH}, 43210$ \\ email: siverd,kstanek, pogge,gaudi, depoy@astronomy.ohio-state.edu \\ ${ }^{2}$ Vanderbilt University \\ 6301 Stevenson Center \\ Nashville, TN 37235 \\ email: joshua.pepper@vanderbilt.edu
}

\begin{abstract}
The Kilodegree Extremely Little Telescope (KELT) is a wide-field $\left(26^{\circ} \times 26^{\circ}\right)$ robotic survey telescope currently operating in Sonoita, Arizona. Assembled from commercial and offthe-shelf devices, KELT currently surveys $\sim 40 \%$ of the Northern sky with sufficient precision to detect transiting planets around bright $(8<V<12)$ stars. In the past several years of operation, over 30,000 science images have been acquired. Planet candidate selection and follow-up are currently underway. A brief overview of past and present survey operations, the data reduction pipeline, and initial results follows below.
\end{abstract}

\section{Science Goals and Current Status}

Following the design guidelines of Pepper et al. (2003), KELT was assembled specifically to find the transiting planets around bright $(8<V<10)$ stars, a region of parameter space that has yet to be explored by most current radial velocity and transit surveys. In addition to the many inherent advantages of transiting systems, the brightest systems lend themselves well to high precision follow-up and thus more sophisticated science programs.

With its current survey parameters, KELT has been fully operational since mid 2006 and has acquired roughly 30,000 science images. At present, full-scale data reduction and candidate selection are underway.

\section{Survey Hardware}

The KELT survey instrument consists of a collection of commercially-available equipment, chosen to meet the requirements of Pepper et al. (2003). The Apogee AP16E camera is a thermo-electrically cooled CCD device with $4 \mathrm{~K} \times 4 \mathrm{~K} 9 \mu \mathrm{m}$ pixels, $15 \mathrm{e}^{-1}$ read noise, and a gain of 3.6. Used in conjunction with a Mamiya 645 medium-format camera lens (42mm aperture, f/1.9) and Kodak Wratten \#8 red-pass filter, this system achieves a wide field of view $\left(\sim 26^{\circ}\right.$ on a side $)$ at $23 \% /$ pixel.

This optical system is mounted atop a Paramount ME robotic German Equatorial mount from Software Bisque. Accompanying software programs (TheSky 6 Professional, CCDSoft, and TPoint), also from Software Bisque, provide script-accessible interfaces 


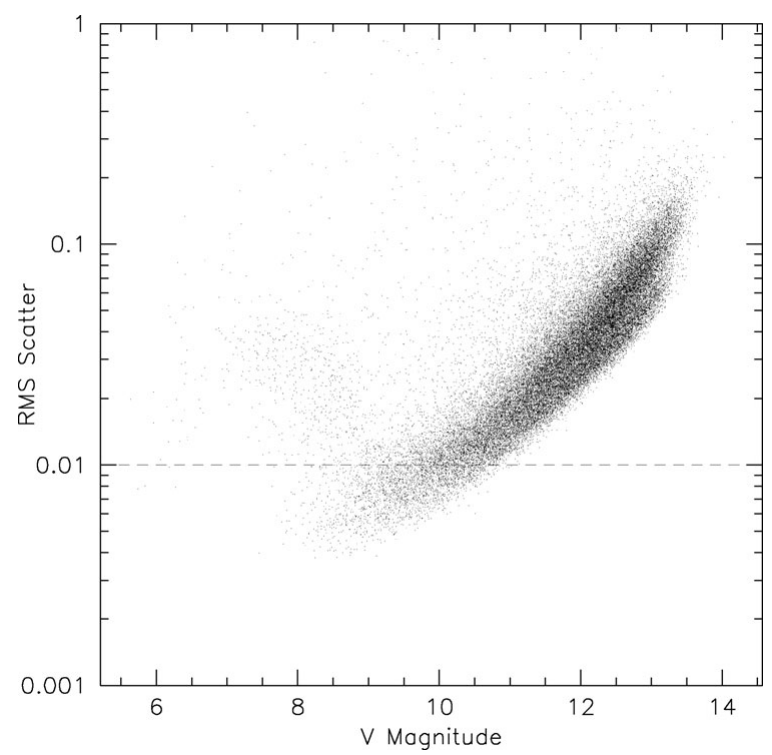

Figure 1. RMS magnitude scatter is shown for a range of approximate V-band magnitudes. Lightcurves shown have approximately 500 data points each. Without detrending, roughly 2500 lightcurves lie below the nominal Jupiter-sized planet detection threshold of $1 \%$ (dashed line). Image vignetting (nearly $30 \%$ center-to-edge) is largely responsible for the vertical spread.

to both camera and mount which enable robotic operation via our control computer running Windows XP Professional. The survey instrument is permanently mounted on a fixed pier at Winer Observatory in Sonoita, AZ. Using a TPoint-generated pointing model, KELT achieves repeatable 3-pixel (1 arcminute) RMS pointing across the entire sky.

\section{Operation and Logistics}

Survey operation is fully unattended. The observing routine, written in VBScript, is responsible for telescope movement, image (dark, flat \& science) acquisition, and preliminary image analysis plus quality control.

Our survey targets 13 star fields at $31.7^{\circ}$ declination (survey site latitude) spaced evenly through 24 hours R.A with slight overlap. By cycling through the (typically 5 or 6) observable fields, KELT achieves good time sampling while minimizing the effects of correlated noise. Improved star sampling and survey bright limit extension are simultaneously achieved with the lens slightly out of focus. Current exposure time is 150 seconds.

After acquisition, images are stored on external USB hard disks. When full, disks are transferred to Ohio State via FedEx in special padded containers. At Ohio State, images are stored on a high-availability, high-speed storage server tuned to facilitate a distributed parallel data reduction scheme.

\section{Data Reduction and Early Results}

To generate lightcurves, KELT relies on a the ISIS image subtraction package (Alard \& Lupton 1998). Although highly effective, these subtraction procedures are highly 


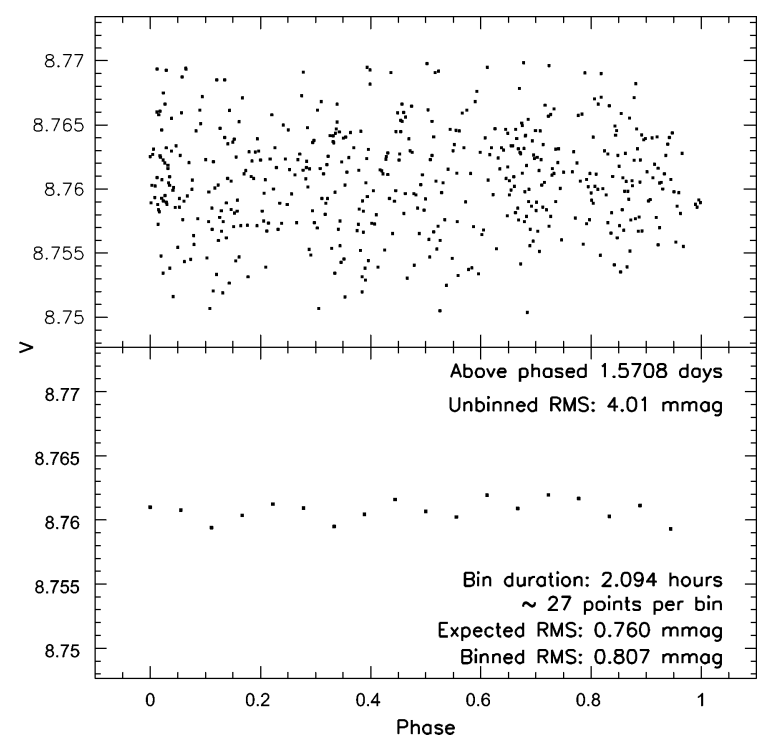

Figure 2. One of the brightest lightcurves from initial data reduction is reproduced above to illustrate KELT survey noise properties. The lightcurve was phased at roughly 1.6 days then binned over $\sim 2.1$ hours. The observed unbinned RMS (4 mmag) falls nearly as $\sqrt{N}$ (to $0.81 \mathrm{mmag}$ ), nearly reaching the correlated-noise-free expected value of $0.76 \mathrm{mmag}$. A correlated residual is apparent at low amplitude. For reference, 2.1 hours is the expected equatorial transit duration for a planet with a 1.6-day period orbiting a solar-type star. Owing to gaps in this particular data set, phasing with a longer period was not possible for this demonstration.

computer-intensive. For KELT, the reduction time of a single image on a typical PC is longer than the mean time between exposures (year-averaged). To make this system tractable, the stock ISIS scripts have been modified to facilitate distributed image reduction across an arbitrary (server-limited) number of computers in parallel. Running ISIS in this distributed parallel fashion (at present $\sim 10$ computers) permits thorough exploration of reduction parameters, which would otherwise be impossible.

Promising early results suggest that many lightcurves $(\sim 2500+$ per field $)$ have sufficient precision to detect Jupiter-sized planets before detrending (Figure 1). Initial tests also indicate that correlated noise has a minimal effect (Figure 2). For pipeline testing, lightcurves of known variables were assembled independently with aperture photometry (Figure 3). These results were compared to those acquired with ISIS and have convincingly demonstrated both the precision and accuracy of ISIS-based lightcurve extraction. 


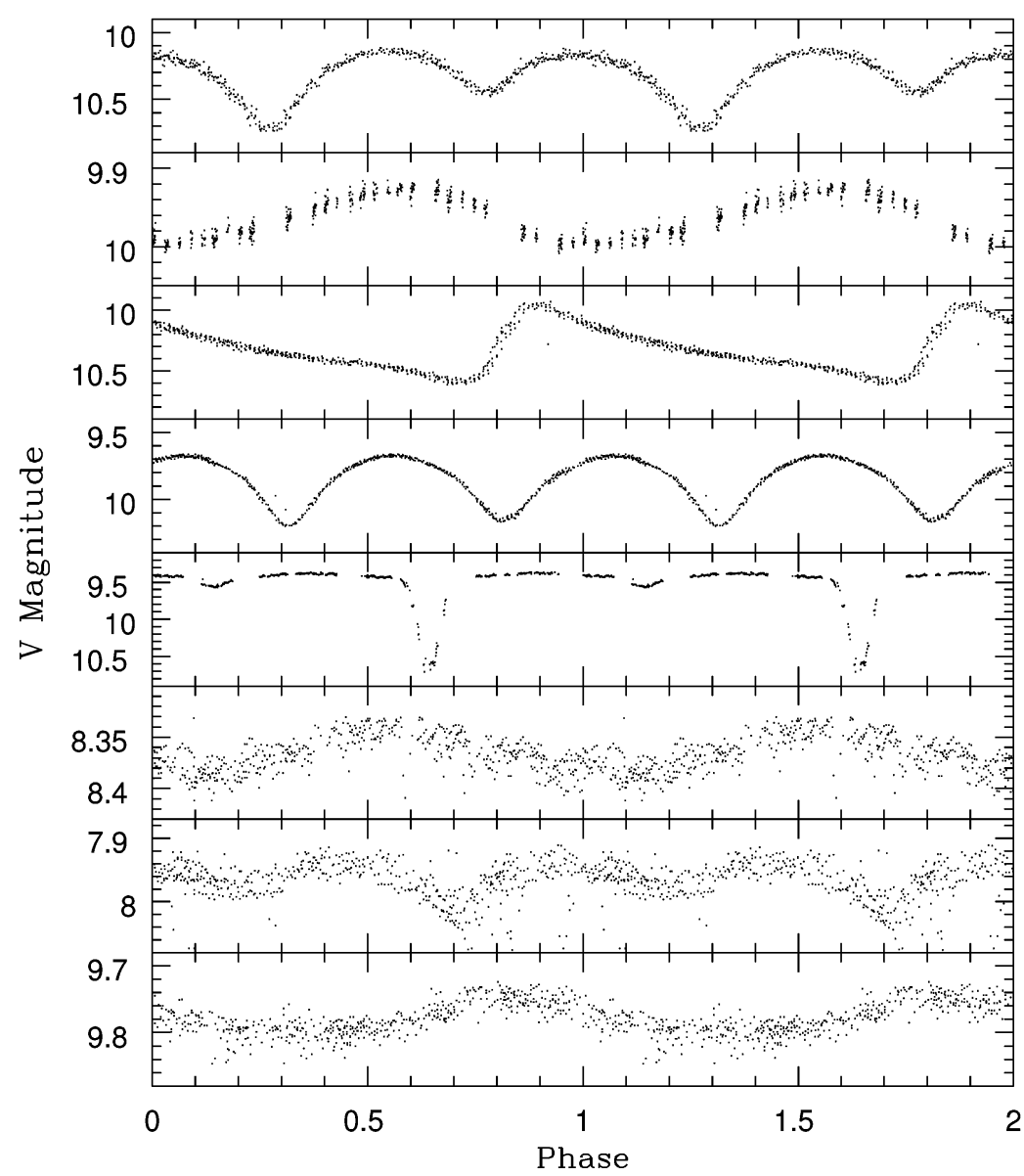

Period

(days)

0.4627

11.63

0.4421

0.5784

4.12

0.861

0.584

0.723

Figure 3. The above lightcurves are a subset of the known variables used for early data reduction and testing (before detrending and extremum clipping). V-band magnitudes are approximate and reflect the average offset between the $\mathrm{V}$ and KELT passpands.

\section{References}

Alard, C. \& Lupton, R. H. 1998, ApJ, 503, 325

Pepper, J., Gould, A., \& Depoy, D. L. 2003, AcA, 53, 213

Pepper, J., Stanek, K. Z., Pogge, R. W. , Latham, D. W., DePoy, D. L., Siverd, R., Poindexter, S., \& Sivakoff, G. R. 2008, AJ, 135, 907

Pepper, J., Pogge, R. W., DePoy, D. L., Marshall, J. L., Stanek, K. Z., Stutz, A. M., Poindexter, S., Siverd, R., O'Brien, T. P., Trueblood, M., \& Trueblood, P. 2007, PASP 119, 923 